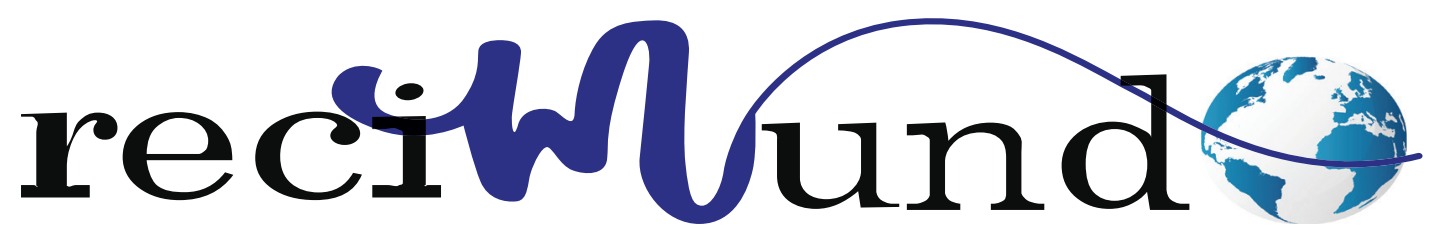

Revista Científica Mundo de la Investigación y el Conocimiento

DOI: 10.26820/recimundo/5.(2).abril.2021.194-202

URL: https://recimundo.com/index.php/es/article/view/1054

EDITORIAL: Saberes del Conocimiento

REVISTA: RECIMUNDO

ISSN: 2588-073X

TIPO DE INVESTIGACIÓN: Artículo de revisión

Código UNESCO: 32 Ciencias Médicas

PAGINAS: 194-202

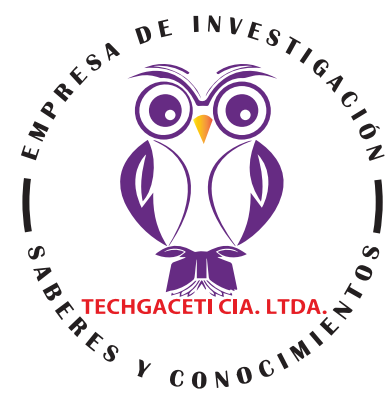

\title{
Utilidad de la pH-metría en el reflujo gastroesofágico
}

Usefulness of pH-metry in gastroesophageal reflux Utilidade da medição de pH-metria no refluxo gastroesofágico

Hilda Margarita Campoverde Chiquito1; Gonzalo Mauricio Yagual Lucas²; Astrid Carolina Haro Parra3;

Ronald Adrián Valencia Rodríguez ${ }^{4}$

RECIBIDO: 15/01/2021 ACEPTADO: 20/03/2021 PUBLICADO: 01/04/2021

1. Médico General en Funciones Hospitalarias; Hospital Monte Siani; Médico Residente Hospital Clínica Kennedy; Guayaquil, Ecuador; hcampoverde1980@hotmail.com; (iD https://orcid.org/0000-0001-6132-0620

2. Médico General en Funciones Hospitalarias; Médico Residente de Cuidados intensivos - Hospital General Monte Sinai; Médico Residente Medicina Interna en Clínica Surhospital; Guayaquil, Ecuador; mauricioyagual68@gmail.com; (iD https:// orcid.org/0000-0003-0966-1369

3. Médico General en Funciones Hospitalarias; Médico Residente de Emergencia - Hospital General Monte Sinaí; Guayaquil, Ecuador; k-ritoharo@hotmail.com; (iD https://orcid.org/0000-0003-2649-4689

4. Médico General en Funciones Hospitalarias; Médico Residente de la Unidad de Cuidados Intensivos; Guayaquil, Ecuador; ronald1930@hotmail.es; (iD https://orcid.org/0000-0003-0730-3701

\section{CORRESPONDENCIA}

Hilda Margarita Campoverde Chiquito

hcampoverde1980@hotmail.com

\section{Guayaquil, Ecuador}

() RECIMUNDO; Editorial Saberes del Conocimiento, 2021 


\section{RESUMEN}

El reflujo gastroesofágico (RGE) es el paso del contenido gástrico hacia el esófago con o sin vómitos o regurgitación, esta última se diferencia del vómito porque se realiza sin esfuerzo y no se presenta en forma explosiva, aunque a veces en el lactante menor puede ser muy intensa. El RGE es un fenómeno fisiológico que ocurre en todas las edades, pero principalmente en el lactante, en que la mayoría de los episodios de RGE duran menos de tres minutos, ocurren en período post prandial y cursan con pocos o ningún síntoma. Existe el criterio generalizado entre los médicos, incluidos los gastroenterólogos, que el diagnóstico del reflujo gastroesofágico (RGE) patológico puede formularse en la mayoría de los casos en base al cuadro clínico, particularmente por la presencia de los síntomas clásicos de pirosis y regurgitación. Sin embargo. Aun cuando el «gold standard» para el diagnóstico de reflujo ácido patológico hacia el esófago, es el estudio de $\mathrm{pH}$ de $24 \mathrm{~h}$. Se considera que los síntomas son molestos y alteran la calidad de vida cuando ocurren dos o más veces por semana. Es una entidad muy prevalente en la población general. En Japón afecta del 6,5 al 9,5\% de la población, en EE.UU. 10\% a 20\%, Europa cercano a 10\% y 20\% y en América Latina del 11,9 al 31,3\%. Se estima que su incidencia es del 4,5-5,4/1000 pacientes por año. La metodología de la investigación, es una revisión de tipo bibliográfico, que se ha apoyado en medios electrónicos como Google Académico, PubMed, entre otros, para la obtención de la información. La hpmetria es una de más mejores técnicas para el manejo de pacientes con RGE, que, junto con el tratamiento médico, han logrado la disminución del tratamiento quirico. La utilidad de la hpmetria está más que demostrada, ya que: permite evaluar la cantidad de relajaciones transitorias que ocurren en el esfínter esofágico inferior, es útil para evaluar la respuesta a la terapia anti secretora, permite determinar la asociación temporal entre el reflujo ácido y frecuencia de un síntoma como tos y en los asmáticos o sibilantes, detectar reflujo ácido que puede ser un factor agravante de sus crisis, se utiliza fundamentalmente para confirmar la sospecha de RGE en pacientes que no responden al tratamiento empírico y presentan endoscopia normal, entre otras.

Palabras clave: Endoscopia, pH-metría, gastroesofágico, vómitos, quirúrgico.

\section{ABSTRACT}

Gastroesophageal reflux (GER) is the passage of gastric contents into the esophagus with or without vomiting or regurgitation, the latter differs from vomiting because it is carried out without effort and does not appear explosively, although sometimes in the young infant it can be very intense. GER is a physiological phenomenon that occurs at all ages, but mainly in infants, in which most episodes of GER last less than three minutes, occur in the post-prandial period, and have few or no symptoms. There is a generalized opinion among physicians, including gastroenterologists, that the diagnosis of pathological gastroesophageal reflux (GER) can be made in most cases based on the clinical picture, particularly by the presence of the classic symptoms of heartburn and regurgitation. However. Even though the "gold standard" for the diagnosis of pathological acid reflux into the esophagus is the 24-hour pH study. Symptoms are considered bothersome and disrupt quality of life when they occur two or more times a week. It is a very prevalent entity in the general population. In Japan it affects 6.5 to $9.5 \%$ of the population, in the US $10 \%$ to $20 \%$, Europe close to $10 \%$ and $20 \%$ and in Latin America from 11.9 to $31.3 \%$. Its incidence is estimated to be 4.5-5.4 / 1000 patients per year. The research methodology is a bibliographic review, which has been supported by electronic media such as Google Scholar, PubMed, among others, to obtain the information. Hypmetry is one of the best techniques for the management of patients with GER, which, together with medical treatment, have achieved a reduction in surgical treatment. The usefulness of hpmetry is more than demonstrated, since: it allows to evaluate the amount of transient relaxations that occur in the lower esophageal sphincter, it is useful to evaluate the response to anti-secretory therapy, it allows determining the temporal association between acid reflux and frequency of a symptom such as cough and in asthmatics or wheezing, detecting acid reflux, which can be an aggravating factor in their attacks, is mainly used to confirm the suspicion of GER in patients who do not respond to empirical treatment and have normal endoscopy, among others.

Keywords: Endoscopy, pH-metry, gastroesophageal, vomiting, surgical.

\section{RESUMO}

O refluxo gastroesofágico (RGE) é a passagem do conteúdo gástrico para o esôfago com ou sem vômito ou regurgitação, este último difere do vômito porque é realizado sem esforço e não parece explosivo, embora às vezes no recém-nascido possa ser muito intenso. RGE é um fenômeno fisiológico que ocorre em todas as idades, mas principalmente em bebês, nos quais a maioria dos episódios de RGE duram menos de três minutos, ocorrem no período pós-prandial, e têm poucos ou nenhuns sintomas. Há uma opinião generalizada entre os médicos, incluindo gastroenterologistas, de que o diagnóstico de refluxo gastroesofágico patológico (RGE) pode ser feito na maioria dos casos com base no quadro clínico, particularmente pela presença dos sintomas clássicos de azia e regurgitação. No entanto, o diagnóstico é feito com base no quadro clínico. Mesmo que o "padrão ouro" para o diagnóstico de refluxo ácido patológico no esôfago seja o estudo de $\mathrm{pH} 24$ horas. Os sintomas são considerados incômodos e perturbam a qualidade de vida quando ocorrem duas ou mais vezes por semana. É uma entidade muito prevalente na população em geral. No Japão afeta de 6,5 a 9,5\% da população, nos EUA de 10\% a 20\%, na Europa perto de 10\% e 20\% e na América Latina de 11,9 a 31,3\%. Sua incidência é estimada em 4,5-5,4 / 1000 pacientes por ano. A metodologia de pesquisa é uma revisão bibliográfica, que tem sido apoiada por meios eletrônicos como o Google Scholar, PubMed, entre outros, para obter as informações. A hipmetria é uma das melhores técnicas para o manejo de pacientes com RGE, que, juntamente com o tratamento médico, conseguiram uma redução no tratamento cirúrgico. A utilidade da hpmetria é mais do que demonstrada, pois: ela permite avaliar a quantidade de relaxões transitórias que ocorrem no esfíncter esofágico inferior, é útil para avaliar a resposta à terapia anti-secreção, permite determinar a associação temporal entre o refluxo ácido e a freqüência de um sintoma como a tosse e em asmáticos ou sibilos, detectar o refluxo ácido, que pode ser um fator agravante em seus ataques, é utilizada principalmente para confirmar a suspeita de RGE em pacientes que não respondem ao tratamento empírico e têm endoscopia normal, entre outros.

Palavras-chave: Endoscopia, pH-metria, gastroesofágico, vômitos, cirúrgico. 


\section{Introducción}

El reflujo gastroesofágico (RGE) es el paso del contenido gástrico hacia el esófago con o sin vómitos o regurgitación, esta última se diferencia del vómito porque se realiza sin esfuerzo y no se presenta en forma explosiva, aunque a veces en el lactante menor puede ser muy intensa. El RGE es un fenómeno fisiológico que ocurre en todas las edades, pero principalmente en el lactante, en que la mayoría de los episodios de RGE duran menos de tres minutos, ocurren en período post prandial y cursan con pocos o ningún síntoma. La regurgitación se produce a diario en el $50 \%$ de los niños menores de tres meses y es el síntoma más visible del RGE madurativo o fisiológico, que generalmente se resuelve espontáneamente en la mayoría de los lactantes sanos a los 12 a 14 meses de edad (Guevara, 2011).

Varios autores han descrito en pacientes con reflujo gastroesofágico (GE) crónico una alteración anatómica de la unión gastroesofágica o cardias, que favorece la presencia y la gravedad de la sintomatología. Este hallazgo anatómico patológico corresponde a una dilatación progresiva del cardias y ha sido denominada por algunos autores como patulous cardia o dilated cardia (Falavigna, Csendes, Henríquez, \& Luengas, 2006, pág. 188).

Se considera que los síntomas son molestos y alteran la calidad de vida cuando ocurren dos o más veces por semana. Es una entidad muy prevalente en la población general. En Japón afecta del 6,5 al 9,5\% de la población, en EE.UU. 10\% a 20\%, Europa cercano a $10 \%$ y $20 \%$ y en América Latina del 11,9 al $31,3 \%$. Se estima que su incidencia es del 4,5-5,4/1000 pacientes por año (Castillo \& Trespalacios, 2015, pág. 431).

Existe el criterio generalizado entre los médicos, incluidos los gastroenterólogos, que el diagnóstico del reflujo gastroesofágico (RGE) patológico puede formularse en la mayoría de los casos en base al cuadro clínico, particularmente por la presencia de los síntomas clásicos de pirosis y regurgitación. Sin embargo. Aun cuando el "gold standard» para el diagnóstico de reflujo ácido patológico hacia el esófago, es el estudio de $\mathrm{pH}$ de 24h. En contraste, la endoscopia se efectúa con mayor frecuencia, a pesar de su limitada capacidad diagnóstica (Csendes, Rencoret, Beltrán, Smok, \& Henríquez, 2004, pág. 20).

En cuanto a la valoración clínica del RGE, la información más reciente muestra la variabilidad de criterios y medios que se utilizan para ese fin. A pesar de eso, la pHmetría esofágica es considerada, en la actualidad, el mejor procedimiento ampliamente disponible para conocer la intensidad y gravedad potencial del RGE, condicionando, en gran medida, su tratamiento (Zarabozo, y otros, 2011, pág. 94).

Actualmente el conocimiento más exhaustivo de la fisiopatología de la ERGE, derivado de la introducción de los estudios funcionales (manometría esofágica y pH-metría), permite afirmar que su patogenia es multifactorial, aceptándose que la ERGE sería el resultado del desequilibrio entre factores defensivos y factores agresivos al nivel de la mucosa esofágica. Así, la barrera antirreflujo con sus diferentes componentes, el aclaramiento esofágico que permite la normalización del pH intraesofágico merced al peristaltismo primario y la resistencia de la mucosa esofágica actuarían como factores defensivos, mientras el ácido refluido desde el estómago y, en menor medida, la pepsina y las sales biliares actuarían como factores agresivos y mediadores principales de la lesión tisular. La ruptura del equilibrio entre factores defensivos y factores agresivos se produciría fundamentalmente por la disfunción de la barrera antirreflujo y en menor medida por la disfunción del aclaramiento esofágico (Arín \& Iglesias, 2003, pág. 252). 


\section{Metodología}

La metodología de la investigación, es una revisión de tipo bibliográfico, que se ha apoyado en medios electrónicos como Google Académico, PubMed, entre otros, para la obtención de la información. La idea fundamental es recolectar la mayor información disponible de otros autores para de esta manera hacer una síntesis acerca del tema de estudio y elaborar un documento lo más completo posible, que sirva de apoyo de otros investigadores.

\section{Resultados}

\section{Diagnostico}

\section{pHmetría}

La monitorización del pH esofágico intraluminal se realiza a través de 1 catéter, de antimonio o fibra de vidrio, de inserción nasal con 1 o más electrodos colocados a lo largo, con el objetivo de capturar, y analizar los eventos de reflujo ácido. Se considera un episodio de reflujo ácido cuando el pH es inferior a 4.0. Los parámetros obtenidos de la pH metría incluyen:

- Número total de episodios de reflujo.

- Número de reflujos mayor a 5 minutos de duración.

- Duración del episodio de reflujo más largo.

El índice de reflujo (IR): porcentaje del total del registro en que el $\mathrm{pH}$ es menor a 4 . Es considerado anormal un IR $>$ a 7 y normal cuando es menor a 3 entre 3 y 7 indeterminado.

\section{Utilidad de la hpmetria}

- La pHmetría permite evaluar la cantidad de relajaciones transitorias que ocurren en el esfínter esofágico inferior, ya que hay una disminución secundaria del $\mathrm{pH}$ por el ascenso del jugo gástrico.
- La pH metría es útil para evaluar la respuesta a la terapia anti secretora.

- Permite determinar la asociación temporal entre el reflujo ácido y frecuencia de un síntoma como tos y en los asmáticos o sibilantes

- Detectar reflujo ácido que puede ser un factor agravante de sus crisis. Sin embargo, adolece de captar los episodios de reflujos no ácidos postprandiales (Guevara, 2011).

- Esta técnica se utiliza fundamentalmente para confirmar la sospecha de RGE en pacientes que no responden al tratamiento empírico y presentan endoscopia normal (Arín \& Iglesias, 2003).

- Pacientes con endoscopia normal que van a ser considerados para cirugía antirreflujo (Arín \& Iglesias, 2003).

- Pacientes en los que se sospecha recidiva del RGE tras cirugía antirreflujo (Arín \& Iglesias, 2003).

- Pacientes con síntomas atípicos (Arín \& Iglesias, 2003).

La pH-metría ambulatoria de 24h es la exploración que ofrece mayor sensibilidad y especificidad para el diagnóstico de la ERGE. Aunque la prueba no proporciona información acerca de la causa del reflujo, ni aporta datos de interés pronóstico, permite cuantificar la exposición al ácido del esófago, analizar su capacidad de aclaramiento, además de dar información cualitativa al poder conocer la asociación de los episodios de reflujo con los síntomas. Hay que tener en cuenta que esta técnica tampoco es lo suficientemente sensible como para considerarla patrón oro en el diagnóstico de la ERGE. Se sabe que hasta un $25 \%$ de los pacientes con esofagitis documentada por endoscopia puede tener un registro pH-métrico normal (Arín \& Iglesias, 2003). 
Tabla 1. Otros tipos de estudio para hpmetria

\begin{tabular}{|c|c|}
\hline Estudio & Descripción \\
\hline $\begin{array}{l}\text { Impedanciometría } \\
\text { intraluminal múltiple } \\
\text { MIl y pH metría }\end{array}$ & $\begin{array}{l}\text { Es un procedimiento que mide los movimientos de fluidos, sólidos y aire } \\
\text { en el esófago. Mide cambios en la impedancia eléctrica (resistencia) } \\
\text { entre los diferentes electrodos colocados a lo largo del catéter esofágico. } \\
\text { Al existir un evento de reflujo hay un cambio de impedancia en el canal } \\
\text { distal del contenido refluido hacia el canal proximal. Se puede calcular } \\
\text { la dirección y la velocidad del bolo, por la distancia definida entre los } \\
\text { canales y el tiempo entre las alteraciones de los patrones de impedancia } \\
\text { de los pares secuenciales de electrodos. Distingue pequeños volúmenes } \\
\text { refluidos y el tipo de contenido. }\end{array}$ \\
\hline $\begin{array}{l}\text { Manometría } \\
\text { esofágica }\end{array}$ & $\begin{array}{l}\text { Mide peristaltismo esofágico, presión de LES y Esfínter esofágico } \\
\text { superior (EES) y la coordinación de estructuras durante la deglución. Ha } \\
\text { tenido un rol importante en identificar a las relajaciones transitorias del } \\
\text { LES como causa de Enfermedad por reflujo (ERGE). Está indicada en: } \\
\text { falla en terapia supresora de ácido, EDA negativa con posible trastorno } \\
\text { de motilidad esofágica, determinar posición de LES (pH), confirmar } \\
\text { diagnóstico de acalasia y otros desórdenes motores. }\end{array}$ \\
\hline $\begin{array}{l}\text { Biopsia y } \\
\text { endoscopia }\end{array}$ & $\begin{array}{l}\text { La endoscopia permite la visualización directa de la mucosa esofágica y } \\
\text { la biopsia la anatomía microscópica de su mucosa Las lesiones } \\
\text { encontradas en la enfermedad por reflujo son: erosiones, con o sin } \\
\text { fibrina, úlceras, estenosis, áreas de posible metaplasia de mucosa } \\
\text { gástrica o intestinal (Barret) y pólipos. También permite hacer el } \\
\text { diagnóstico de anomalías anatómicas que predispongan a RGE } \\
\text { patológico como es la hernia hiatal. }\end{array}$ \\
\hline $\begin{array}{l}\text { Radiografía esófago } \\
\text { estómago duodeno }\end{array}$ & $\begin{array}{l}\text { La Radiografía EED (Rx EED) contrastada con bario, tiene un rol en } \\
\text { detectar alteraciones anatómicas como fistula, mal rotación, estenosis } \\
\text { pilórica, estenosis, acalasia, consideradas diagnóstico diferencial de } \\
\text { RGE. El examen tiene una sensibilidad de } 31-86 \% \text { y especificidad del } \\
21-83 \% \text {. Tiene falsos positivos y falsos negativos, por la corta duración } \\
\text { del examen. No se justifica realizar series radiológicas para el } \\
\text { diagnóstico, ni para evaluar la gravedad del reflujo. }\end{array}$ \\
\hline Cintigrafía & $\begin{array}{l}\text { Esta técnica evalúa la presencia de reflujo post prandial independiente } \\
\text { del PH gástrico y a diferencia de otros exámenes detecta reflujo no } \\
\text { ácido. Brinda información sobre el tiempo del vaciamiento gástrico, el } \\
\text { que puede estar retardado en ERGE. No existe una estandarización en } \\
\text { los valores normales por edad. La Cintigrafía gastroesofágica detecta } \\
\text { episodios de reflujo y aspiración durante un período corto después de la } \\
\text { alimentación. Los episodios de aspiración se pueden detectar a la hora } \\
\text { y hasta las } 24 \text { hrs del inicio del examen, sin embargo, tiene baja } \\
\text { sensibilidad para detectar las micro aspiraciones. Un test negativo, no } \\
\text { excluye la posibilidad que existan aspiraciones. La sensibilidad de este } \\
\text { test es baja ( } 15 \text { a } 59 \% \text { ) y la especificidad es alta ( } 33 \text { a } 100 \% \text { ). }\end{array}$ \\
\hline Ultrasonografía & $\begin{array}{l}\text { Es una técnica no recomendada para el diagnóstico y manejo de RGE } \\
\text { en niños. No brinda mayor información que lo obtenido por otras técnicas } \\
\text { y no tiene correlación con los episodios de RGE detectados por } \\
\text { pHmetría. }\end{array}$ \\
\hline
\end{tabular}

Fuente: (Guevara, 2011). 
Tabla 2. Síntomas de reflujo gastroesofágico

\begin{tabular}{|c|c|}
\hline Síntoma & Descripción \\
\hline Pirosis & $\begin{array}{l}\text { La pirosis (sensación de ardor o quemazón que surge del } \\
\text { estómago y puede irradiarse por el área retroesternal hacia el } \\
\text { cuello) se considera el síntoma más común de la ERGE. No } \\
\text { obstante, la ausencia de un patrón oro para el diagnóstico de la } \\
\text { enfermedad, como se ha comentado con anterioridad, no permite } \\
\text { conocer con certeza la capacidad predictiva de este síntoma. La } \\
\text { pirosis aparece unos } 30-60 \text { minutos después de la ingesta y suele } \\
\text { aliviarse con la toma de antiácidos, aunque sea sólo de forma } \\
\text { transitoria. Cuando es muy intensa el paciente puede percibirla } \\
\text { como dolor en epigastrio o bien a nivel retroesternal. }\end{array}$ \\
\hline Regurgitación & $\begin{array}{l}\text { La regurgitación ácida es un síntoma muy específico de ERGE, } \\
\text { aunque menos frecuente que la pirosis. Consiste en el paso de } \\
\text { material contenido en el estómago a la boca, bien de forma } \\
\text { espontánea o desencadenado por determinadas posturas que } \\
\text { aumentan la presión intraabdominal. Es típico que aparezca al } \\
\text { inclinarse hacia delante o en decúbito lateral derecho. Cuando se } \\
\text { producen episodios de regurgitación nocturna, pueden } \\
\text { manifestarse en el paciente en forma de disnea o de crisis de tos } \\
\text { de tipo irritativo. }\end{array}$ \\
\hline Disfagia & $\begin{array}{l}\text { Se considera como disfagia, la sensación de que el alimento se } \\
\text { detiene en su paso desde la boca hasta el estómago. En la ERGE, } \\
\text { la disfagia puede deberse a una alteración de la motilidad } \\
\text { esofágica (hipoperistaltismo esofágico que dificulta la progresión } \\
\text { del bolo alimenticio) o a la existencia de una lesión orgánica, bien } \\
\text { sea una esofagitis o una estenosis péptica. En el primer caso, el } \\
\text { paciente suele presentar una disfagia paradójica (para líquidos } \\
\text { exclusivamente o simultáneamente para sólidos y para líquidos) } \\
\text { mientras que cuando hay lesiones esofágicas, la disfagia es } \\
\text { progresiva, inicialmente para sólidos y posteriormente para } \\
\text { líquidos. }\end{array}$ \\
\hline Odinofagia & $\begin{array}{l}\text { La odinofagia se define como una deglución dolorosa a nivel } \\
\text { retroesternal y hay que señalar, que no es un síntoma habitual de } \\
\text { la ERGE. Habitualmente la odinofagia se manifiesta por un dolor } \\
\text { de intensidad leve, aunque en determinadas circunstancias, } \\
\text { puede ser intenso, dificultando incluso la alimentación del } \\
\text { paciente. Su presencia suele indicar bien la existencia de una } \\
\text { esofagitis, generalmente grave, o bien la aparición de } \\
\text { contracciones sincrónicas o terciarias y es indicación de realizar } \\
\text { una endoscopia. }\end{array}$ \\
\hline
\end{tabular}

Fuente: (Arín \& Iglesias, 2003) 


\section{Tratamiento}

Los objetivos del tratamiento son: aliviar los síntomas; asegurar una relación peso/talla adecuada; mejorar la inflamación producida por el reflujo ácido o la esofagitis, que no siempre es secundaria a reflujo ácido, también puede ser por reflujo alcalino; y prevenir las complicaciones respiratorias y otras, derivadas del reflujo crónico. Las herramientas disponibles para tratar el RGE son de tipo médico, farmacológico y quirúrgico. En la actualidad, con la aparición de los inhibidores de la bomba de protones han disminuido los casos en que se indica tratamiento quirúrgico, pero esta familia de fármacos también presenta complicaciones a largo plazo, desde un punto de vista teórico, como la hipergastrinemia y posibles alteraciones celulares (Venegas, 2006).

El tratamiento médico depende de la edad del paciente. En el niño mayor, los estilos de vida son importantes; por ejemplo, los adolescentes tienen ritmos especiales de comida y de vida. Afortunadamente, hoy se usan los pantalones bajo la cintura y ya no aumenta la presión intraabdominal con el cinturón, lo que favorecía el reflujo, pero también está de moda trasnochar, consumir alcohol en forma excesiva, ingerir comidas grasas, que causan estasis gástrica y fumar, que también aumenta la retención gástrica (Venegas, 2006).

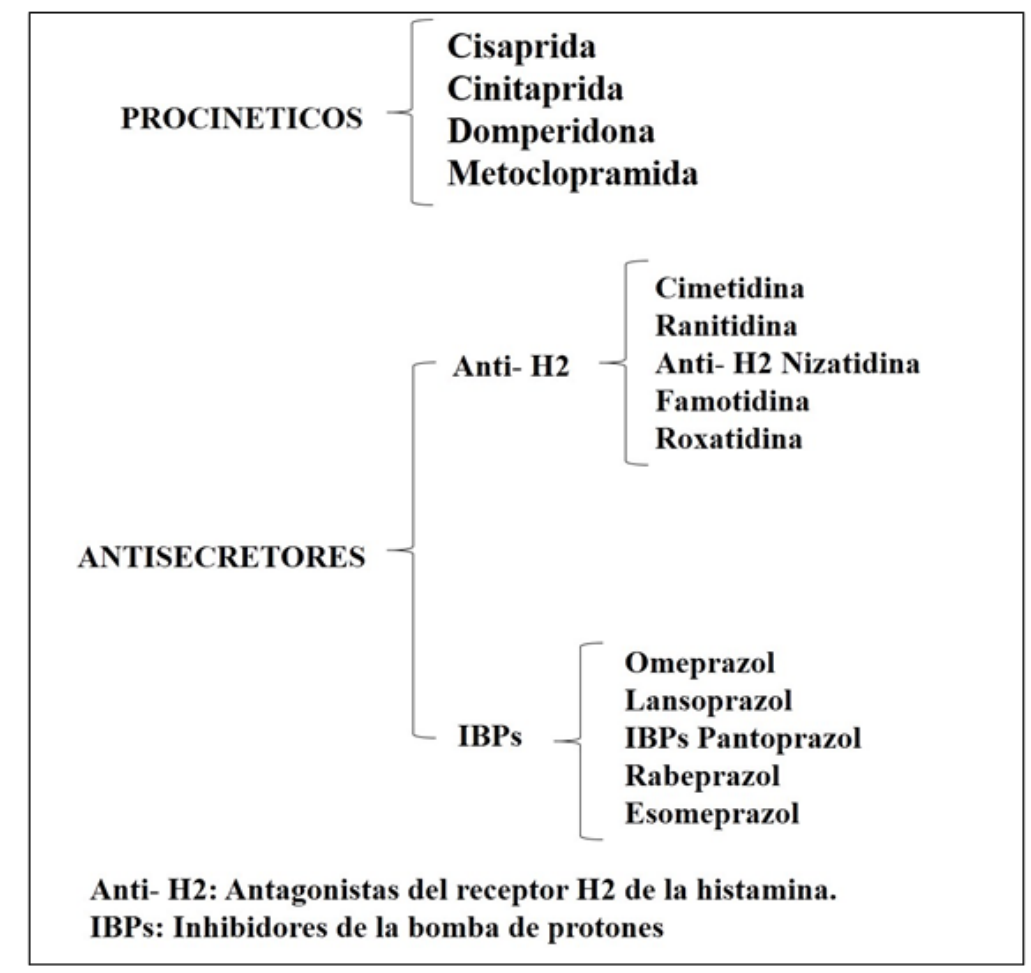

Imagen 1. Grupos farmacológicos en el tratamiento de la ERGE

Fuente: (Arín \& Iglesias, 2003)

Respecto al tratamiento postural, se sabe que la posición semisentada no es la mejor, porque conlleva más irritabilidad y llanto, menos tiempo de sueño y mayor exposición de la mucosa esofágica a la acidez, si se la compara con la posición prona simple o anti Trendelenburg de 30 grados. En general, se recomienda la posición supina corriente. A principios de 1990, el registro de $\mathrm{pH}$ esofágico demostró que los lactantes puestos en posición decúbito prono para dormir tenían menos exposición al reflujo (Venegas, 2006). 


\subsection{Tratamiento quirúrgico}

El tratamiento quirúrgico está dirigido a reconstruir la barrera antirreflujo que se encuentra alterada en los pacientes afecta-dos por esta enfermedad. Desde el punto de vista patogénico, el tratamiento con cirugía antirreflujo resulta más lógico que el tratamiento médico, dado que es en la disfunción de la barrera donde subyace el mecanismo patogénico principal de la enfermedad. La técnica quirúrgica universalmente aceptada es la funduplicatura, con sus diferentes variantes (total, parcial, etc.). El abordaje puede realizarse por cirugía abierta o por vía laparoscópica, técnica que ha adquirido gran predicamento en los últimos años, dado que, entre otras ventajas, reduce significativamente la estancia hospitalaria.

Son indicaciones del tratamiento quirúrgico:

- La recidiva precoz en un paciente joven que responde al tratamiento médico y desecha el tratamiento farmacológico de por vida.

- El fracaso del tratamiento médico con persistencia de los síntomas o de las lesiones endoscópicas, aunque el paciente esté asintomático, así como la existencia de complicaciones frecuentes (hemorrágicas, respiratorias, etc.).

- La intolerancia al tratamiento farmacológico (Arín \& Iglesias, 2003).

\section{Complicaciones}

Numerosas complicaciones han sido asociadas con la ERGE incluyendo la esofagitis erosiva, las estenosis y el esófago de Barrett. La obesidad ha mostrado ser un factor de riesgo de enfermedad erosiva, esófago de Barrett y adenocarcinoma. Las esofagitis severas son más comunes en los ancianos y en general existe recaída si no se instituye un tratamiento de mantenimiento (Olmos, Piskorz, \& Vela, 2016).
Las estenosis pépticas son infrecuentes dado el uso generalizado de los tratamientos antisecretores. Las estenosis tienden a ocurrir más frecuentemente en pacientes ancianos y con una larga duración de síntomas no tratados en el contexto de una motilidad esofágica anormal. El esófago de Barrett es la única complicación de la ERGE con potencial de malignidad. El esófago de Barrett se encuentra en el 5 al $15 \%$ de los pacientes a los que se les realiza endoscopía por síntomas de reflujo. Las guías actuales recomiendan que los pacientes con Barrett endoscópico confirmado deben ser enrolados en programas de vigilancia en búsqueda de displasia (Olmos, Piskorz, \& Vela, 2016).

\section{Conclusiones}

La hpmetria es una de más mejores técnicas para el manejo de pacientes con RGE, que, junto con el tratamiento médico, han logrado la disminución del tratamiento quirico. Este último solo es empleado o sugerido en base a la gravedad de la patología. Todos los test e índices hpmetricos excepto el numero total de reflujos son los discriminantes entre pacientes operados y no operados.

La utilidad de la hpmetria esta mas que demostrada, ya que: permite evaluar la cantidad de relajaciones transitorias que ocurren en el esfínter esofágico inferior, es útil para evaluar la respuesta a la terapia anti secretora, permite determinar la asociación temporal entre el reflujo ácido y frecuencia de un síntoma como tos y en los asmáticos o sibilantes, detectar reflujo ácido que puede ser un factor agravante de sus crisis, se utiliza fundamentalmente para confirmar la sospecha de RGE en pacientes que no responden al tratamiento empírico y presentan endoscopia normal, entre otras.

De igual manera la valoración inicial que se haga del paciente es fundamental para la indicación del tratamiento, ya que dicha va-




loración no se puede sustentar solo en la sintomatología, a la hora de la indicación de alguna terapia. Es por ello que la hpmetria es la mejor opción ya que mide si hay o no reflujo ácido patológico, en contraste con la endoscopía que no demuestra presencia de reflujo gastroesofágico y sólo busca encontrar algún daño en la mucosa esofágica y poder tomar biopsias.

La evolución de la medicina y la fisiopatología de la RGE han logrado establecer métodos de diagnósticos más rigurosos (que han permitido descubrir que esta patología se genera por múltiples factores), para poder clasificar adecuadamente a los pacientes y así poder descartar patologías diferentes al reflujo gastroesofágico.

\section{Bibliografía}

Arín, A., \& Iglesias, M. (2003). Arín, A., \& Iglesias, M. R. (2003, August). Enfermedad por reflujo gastroesofágico. In Anales del sistema sanitario de Navarra. Gobierno de Navarra. Departamento de Salud, 2(26), 251-268.

Castillo, R. O., \& Trespalacios, A. (2015). Impacto de las medidas generales en el tratamiento del reflujo gastroesofágico: una revisión basada en la evidencia. Revista Colombiana de Gastroenterología, 30(4), 431-446.
Csendes, A., Rencoret, G., Beltrán, M., Smok, G., \& Henríquez, A. (2004). Correlación entre síntomas de reflujo gastroesofágico y resultados de la pHmetría de 24 horas en pacientes con estudio endoscópico normal o levemente alterado. Revista médica de Chile, 132(1), 19-25.

Falavigna, M., Csendes, A., Henríquez, A., \& Luengas, R. (2006). Comparación entre el aspecto endoscópico del cardias, hallazgos manométricos y pHmetría de 24 horas en pacientes con síntomas de reflujo gastroesofágico crónico. Revista médica de Chile, 134(2), 187-192.

Guevara, G. (2011). Reflujo gastroesofágico en pediatría. Revista chilena de pediatría, 82(2), 142149.

Olmos, J. A., Piskorz, M., \& Vela, M. (2016). Revisión sobre enfermedad por reflujo gastroesofágico (ERGE). Acta Gastroenterológica Latinoamericana, 46(2), 160-172.

Venegas, G. (2006). Tratamiento médico del reflujo gastroesofágico. Medwave, 6(7).

Zarabozo, E. E., Hurtado, C., Sánchez, E., Muñoz, I., Valero, S., \& Velasco, R. (2011). Enfermedad por reflujo gastroesofágico. Valoración e indicación de tratamiento quirúrgico mediante pHmetría esofágica. Cir Pediatr, 24, 93-97.

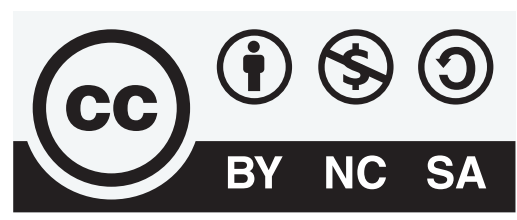

CREATIVE COMMONS RECONOCIMIENTO-NOCOMERCIAL-COMPARTIRIGUAL 4.0.

\section{CITAR ESTE ARTICULO:}

Campoverde Chiquito, H. M., Yagual Lucas, G. M., Haro Parra, A. C., \& Valencia Rodríguez, R. A. (2021). Utilidad de la pH-metría en el reflujo gastroesofágico. RECIMUNDO, 5(2), 194-202. https://doi.org/10.26820/recimundo/5.(2).

abril.2021.194-202 